\title{
ESPACIO TRASFRONTERIZO ENTRE BELICE Y MÉXICO A PRINCIPIOS DEL SIGLO XX. ANÁLISIS SOCIO-DEMOGRÁFICO DEL IMPACTO DE LA MIGRACIÓN EN LA FORMACIÓN FAMILIAR
}

\author{
Allan Ortega Muñoz \\ Instituto Nacional de Antropologia e Historia \\ Centro INAH Quintana Roo \\ allan_ortega@inah.gob.mx
}

\section{RESUMEN}

Belice y México comparten características demográficas, culturales y económicas. Su gente fronteriza ha tenido procesos de sociabilidad, impactando en la formación de sus familias y su modo de asimilación a los lugares de destino al momento de migrar. Se evaluaron estos procesos a través de los registros civiles de nacimientos y defunciones de Corozal, Belice y del sur de Quintana Roo, México (1885 a 1955), con la finalidad de reconstruir las familias, de ahí se obtuvo información sobre su fecundidad y tipos de familias (endo/exogámicas). Los resultados muestran diferencias en estos rubros, por lo que cada grupo social (cultura íntima) vive diferente su proceso migratorio.

Palabras clave: frontera México-Belice, formación familiar, relaciones interétnicas, migración. 


\title{
TRANSBORDER SPACE BETWEEN BELIZE AND MEXICO IN THE EARLY 20TH CENTURY: A SOCIO-DEMOGRAPHIC ANALYSIS OF THE IMPACT OF MIGRATION ON FAMILY FORMATION
}

\begin{abstract}
Belize and Mexico share demographic, cultural and economic characteristics. Their bordering peoples have experienced sociability processes that have an impact on family formation and their mode of assimilation to their destinations when they migrate. These processes were evaluated through the civil registry of birth and death records in Corozal, Belize and South Quintana Roo, Mexico (from 1885 to 1955) with the purpose of reconstructing family composition. Information about fertility and family types (endo/exogamic families) was drawn from the same source. Results show differences in these areas. Each social group (intimate culture) thus has a different experience of its migratory process.
\end{abstract}

Key words: Mexico-Belize border, family formation, inter-ethnic relations, migration. 


\section{INTRODUCCIÓN}

Belice, Honduras Británica antes de 1981 (Hubbard 2000: 52, Toussaint 1993: 109), es un país conformado por un crisol de grupos sociales provenientes de diferentes partes del mundo. La población se encuentra segregada por regiones con rasgos históricos que la confirman como una sociedad de inmigrantes con diferencias culturales delimitadas por tiempos de llegada: al norte del país, en los Distritos de Corozal y Orange Walk, se encuentran asentados los mayas y los mestizos, ambos de origen peninsular yucateco; al sur, en los Distritos de Toledo y Stann Creek, los garínagu con idioma garífuna —indígenas caribes con influencias africanas-; al oriente, en Orange Walk, indígenas mayas quechís y mopanes, con ascendencia centroamericana, y hacia el centro del país, en las ciudades de Belice y Belmopan, se asienta la totalidad de la población criolla, descendientes de esclavos africanos. Estos grupos tienen definido su orden cronológico de llegada al país (Bolland 1992: 276), siendo que a finales del siglo XIX ya estaban los cuatro grupos definidos, apareciendo a principios del siglo $\mathrm{XX}$ pequeños grupos de sirios y menonitas, así como otras minorías, entre ellas la china, llegados en el año 1885 junto con los jamaiquinos y barbarienses (Cal 1992: 4).

Belice está vinculado a un proyecto colonial y con una vida más caribeña que centroamericana, ya que nunca ha sido parte real de la dinámica y las organizaciones regionales centroamericanas. Por otro lado, la mayoría de los estudios de las Indias Occidentales Británicas (West Indies) se han centrado en las islas productoras de azúcar y han excluido virtualmente a Belice, e incluso se ha debatido el hecho de que sea comparable con ellas (Bolland 1992: 18, Cal 1992, Casillas y Castillo 1994: 45-46). No obstante a esta virtual lejanía de Centroamérica y/o el Caribe, con México ha tenido un importante contacto en diversas esferas políticas, demográficas, económicas y culturales, sobre todo en la franja fronteriza.

De estas relaciones es de interés particular las migratorias entre ambos países, fuente de construcción y fortalecimiento de las localidades, sobretodo de la de Quintana Roo a 
principios de siglo XX. Debemos comprender que estas relaciones de intercambio de población se dan en un marco de heterogeneidad cultural, lo que marca las relaciones sociales de estos grupos en el ámbito de identidad. Por lo tanto, cabe pensar que a ambos lados de la frontera sur de México, las dos naciones son naciones políticas, donde la gente a ambos márgenes del río realiza un contrato social con sus instituciones de pertenencia y fidelidad, al menos aparente, y donde los lazos culturales avasallan a los políticos sin llegar a desestabilizar la región.

Retomando la idea anterior, el objetivo del artículo es demostrar que la migración, en un espacio regional trasfronterizo, tiene efectos en la sociabilidad de los diferentes grupos sociales que habitaron entre Belice y México. Para ello, analizo la formación familiar de estos grupos, conglomerados bajo un constructo teórico, el de culturas íntimas (LomnitzAdler 1995) durante los primeros treinta años del siglo XX. Durante este tiempo y espacio determinado, se comienzan a formar diversas localidades del sur de Quintana Roo, entre ellas la actual capital del estado, por lo que se convierte en un laboratorio social para evaluar procesos sociales y demográficos en Quintana Roo como reflejo de lo que también sucede en el lado beliceño.

Las preguntas que orientan mi exposición están en función de la diversidad étnica de la región, por lo que me cuestiono: ¿coexistieron diversos comportamientos demográficos, tantos como diversos grupos sociales se presentaron?, o bien, ¿pudo haberse presentado una convergencia de éstos entre los grupos? Si existió, ¿la convergencia se debió a un proceso de integración y/o asimilación de los migrantes en la sociedad de destino provenientes de Belice, así como de otras partes de México y del mundo? Si no hubo tal convergencia, ¿se debió a que los migrantes mantuvieron actividades trasnacionales, sobre todo para los beliceños, con lo que perpetuaron los comportamientos demográficos que traían consigo, evitando la asimilación? 


\section{LA FRONTERA Y SUS LIMITANTES: LA REGIÓN DE ESTUDIO}

La frontera sur de México, además de dividir a México de Belice geopolíticamente es también un puente de intersecciones entre naciones, entre pueblos, pues «la frontera, al igual que la identidad, tiene múltiples dimensiones, concreciones y prácticas» (Fábregas 1992b: 5). Históricamente se ha dado este proceso de manera natural, por lo que existe una cotidianidad en estos flujos, que van más allá de la conformación como entidad nacional (Belice) y federal (Quintana Roo) de los espacios limítrofes (Casillas y Castillo 1994: 133). Fábregas (1990) señala que lo importante no es el análisis de la situación de frontera reducida a la cuestión territorial entre Estados nacionales o entidades jurídicas diferenciadas, sino la revisión de los procesos históricos y la variada diversidad de las situaciones de frontera.

Para comprender entonces el área bajo estudio considero el sur del actual estado de Quintana Roo, México, y el norte de Belice, como una región (Fábregas 1992a, LomnitzAdler 1995) cuya característica fundamental es el proceso histórico-social de la migración, que tiene un elemento esencial, la frontera natural —el Río Hondo—, donde ocurre un proceso trasfronterizo, entendiéndolo como la continua interacción migratoria, así como de intercambio de recursos y de capital. La migración trasformó las diferentes localidades asentadas al margen de la frontera, pues comparten un mismo proceso cultural y social mediante un proceso de sociabilidad que es «la forma en que las personas se "interconectan" entre sí forjando relaciones entre identidades diferenciadas, subrayando facetas particulares de sus propias identidades y articulándolas con facetas complementarias de las identidades de los otros, lo cual podría hacerse a través de un origen regional compartido» (Kearny 1996, citado por Gledhill 1999: 45). Estos elementos crearon una comunidad con identidad propia, diferente a los dos centros de poder económico, político y social de la península de Yucatán, como eran en ese momento las ciudades de Mérida, Yucatán, en México, y Belice, en Honduras Británica. 
Mapa 1. Región cultural, frontera México-Belice a estudiar durante 1900-1935.

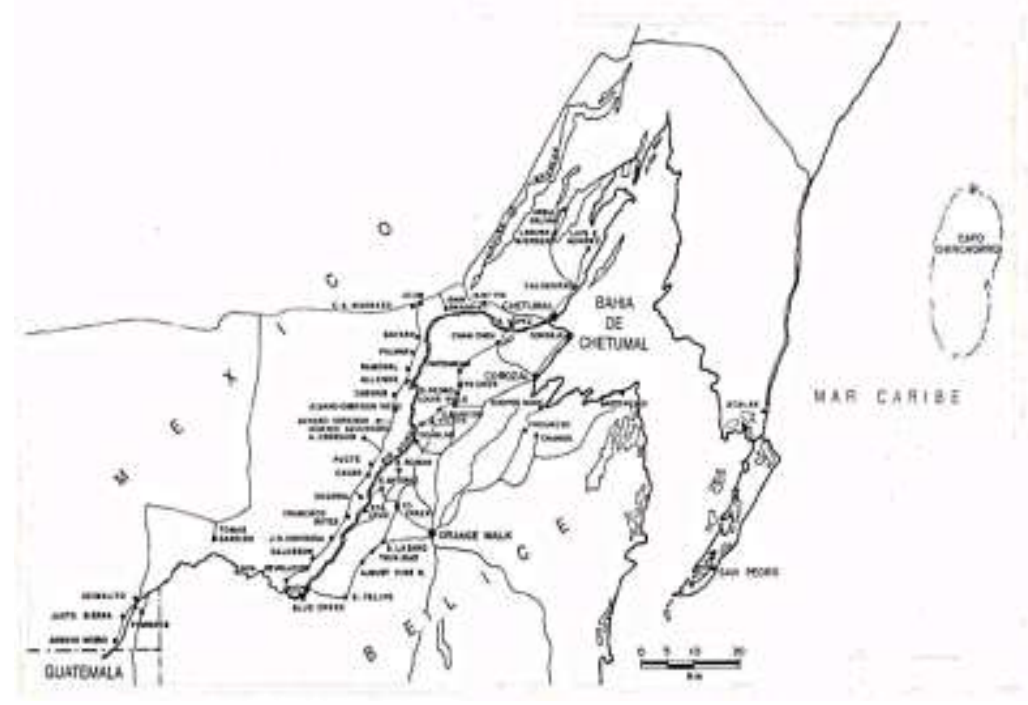

Fuente: Imagen tomada de Arnaiz et al. 1993.

\section{FORMACIÓN FAMILIAR Y CULTURAS ÍNTIMAS}

En la formación familiar hay arreglos entre familias, por lo que existen decisiones no individuales (Bourdieu 1976) en su conformación, aunque a lo largo del tiempo puede cambiar a una decisión más individual (Locoh 1996). Por lo que en la formación familiar o de parejas operan una serie de factores tanto a nivel individual, demográfico y socioeconómico. La combinación de estos factores incide en las normas matrimoniales, y a su vez establece un mercado matrimonial, lo cual permite analizar patrones de nupcialidad —en tiempo y prevalencia—, así como sistemas familiares — tamaño y tipo de familia(Dixon 1971: 221-223, United Nations 1988: 14).

Para poder estudiar la formación familiar entre los diferentes grupos sociales de la región, se empleó un conjunto de manifestaciones reales, regionalmente diferenciadas, de la cultura de clase y de las fronteras étnicas, en un ambiente regional específico, que clasifica a los grupos en culturas íntimas (Lomnitz-Adler 1999: 53). La dinámica o la interacción entre las 
culturas íntimas se da dentro del marco de la cultura de las relaciones sociales, que «es el campo simbólico o lenguaje en el que se establecen objetivamente las relaciones de poder entre culturas íntimas... en un conjunto de marcos institucionales» (Lomnitz-Adler 1995: 46-47, 50). Los marcos interaccionales se construyen por la institucionalización de las relaciones de poder — hegemonía— y la correspondiente resistencia a la misma.

El modelo busca ver la dinámica de la cultura regional: surgen jerarquías de poder tanto dentro como entre los grupos de identidad. Por eso, el análisis de las fronteras sociales requiere de una comprensión histórica de la interacción entre las experiencias culturales compartidas — cultura íntima - y la construcción ideológica de los grupos de identidad —ideología localista — Los ritmos de trasformación de la cultura íntima no son idénticos a la política de la identidad. Pueden surgir nuevas culturas íntimas, y las existentes pueden declinar dentro de un grupo de identidad, mientras este conserva sus mismas fronteras, o pueden surgir culturas íntimas que reclamen para sí una identidad excluyente (LomnitzAdler 1995: 56). En cada momento, al correr el tiempo, hay la coexistencia de culturas íntimas residuales, dominantes y emergentes, así como las correspondientes ideologías localistas (Lomnitz-Adler 1995: 57).

Para comprender estos procesos de sociabilidad, promovidos por la migración bidireccional (Belice-México) entre las culturas íntimas es necesario verlos a través de dos enfoques teóricos de la migración: la asimilación de los grupos migrantes y los procesos trasnacionales, que aquí retomaré como trasterritorialismo (véase Ortega 2006). La asimilación permite entender y describir la integración en la corriente principal de una sociedad por individuos y grupos étnicos migrantes, y se le puede definir como la declinación y la desaparición de una distinción étnica y las diferencias socioculturales que las expresan (Alba y Nee 1999). En la literatura se han descrito diversas dimensiones de ella (Gordon 1964, Masey 1985, Alba y Golden 1986, Massey y Denton 1988, Gans 1992, 1999, Portes y Zhou 1993), que va desde la aculturación hasta la asimilación estructural, 
cuyo indicador esencial son los matrimonios entre grupos que integran la sociedad (Alba y Golden 1986, Gans 1999).

En cuanto al término trasterritorialismo, lo retomo de las propuestas teóricas del trasnacionalismo (Basch, Glick Schiller y SzantonBlanc 1994, Guarnizo y Smith 1999, Portes, Guarnizo y Landolt 1999), el cual permite analizar las ocupaciones y actividades sociales, económicas, políticas del migrante, mismas que son habituales y sostenidas a través del tiempo entre fronteras nacionales y/o geopolíticas diferentes, como es el caso que nos ocupa, entre Belice y México. Por consiguiente, es necesario localizar las diferentes culturas íntimas que se encontraban en la región así como los marcos institucionales, económicos y políticos, bajo los cuales interactuaban, es decir, la búsqueda de la cultura de las relaciones sociales existentes en ellas y analizar cómo cambia, si es que lo hace, a ambos lados de la frontera.

\section{MATERIAL Y MÉTODOS}

Se analizaron los registros civiles de nacimientos y defunciones de las localidades del sur de Quintana Roo, México y las del Distrito de Corozal, al norte de Belice, para los periodos comprendidos entre 1885-1955. Del total de registros de nacimientos (véase Cuadro 1) se identificaron 3,692 madres dentro del periodo de observación (1900-1935), y 2,743 padres varones. De este universo se seleccionó una muestra aleatoria de $10 \%$ de las mujeres que se encontraba dentro de la temporalidad de estudio. Con estos datos se realizaron análisis longitudinales de estos fenómenos demográficos, lo que permitió la reconstrucción familiar de acuerdo con el método desarrollado por L. Henry (Fleury 1985, Henry 1983). Consecuentemente, la reconstrucción de familias fue a partir de 415 madres, obteniéndose una muestra total de 863 madres y 821 padres varones reconstruidos. 
Cuadro 1. Número de eventos registrados por quinquenios en las estadísticas vitales del

Distrito sur de Quintana Roo, México, y Distrito de Corozal, H. B., 1885-1955.

\begin{tabular}{|c|r|r|r|r|}
\hline \multirow{2}{*}{$\begin{array}{c}\text { Quinquenio de } \\
\text { ocurrencia } \\
\text { del evento }\end{array}$} & \multicolumn{2}{|c|}{ Distrito sur de Quintana Roo } & \multicolumn{2}{c|}{ Distrito de Corozal, H.B. } \\
\cline { 2 - 5 } & Nacimientos & Defunciones & Nacimientos & Defunciones \\
\hline $1885-1889$ & & & 1,086 & 1,010 \\
\hline $1890-1894$ & & & 1,276 & 1,456 \\
\hline $1895-1899$ & & & 1,295 & 1,077 \\
\hline $1900-1904$ & 3 & & 1,262 & 973 \\
\hline $1905-1909$ & 65 & 38 & 1,161 & 1,003 \\
\hline $1910-1914$ & 270 & 159 & 1,356 & 885 \\
\hline $1915-1919$ & 344 & 366 & 1,260 & 1,273 \\
\hline $1920-1924$ & 319 & 297 & 1,322 & 969 \\
\hline $1925-1929$ & 583 & 388 & 1,441 & 799 \\
\hline $1930-1934$ & 748 & 33 & 665 & 764 \\
\hline $1935-1939$ & 1,183 & 76 & & \\
\hline $1940-1944$ & 2,187 & 355 & & 10,209 \\
\hline $1945-1949$ & 2,745 & 360 & & \\
\hline $1950-1954$ & 3,116 & 156 & & \\
\hline $1955-1959$ & 511 & 41 & & \\
\hline Total de eventos & 12,074 & 2,269 & 12,124 & \\
\hline
\end{tabular}

Fuente: Estadísticas vitales 1885-1955, Registro Civil de Chetumal, México, y Corozal. H.B. Cálculos propios.

Una vez reconstruidas las familias, a cada una de estas parejas se les ubicó tanto por cohorte de nacimiento de la madre, como dentro de una cultura íntima - Dominante, Emergente, Residual-: el grupo Dominante es aquel que tiene el poder económico y político de la sociedad en la que se desenvuelve, mientras que el grupo Residual es el que tiene y vive en antagonismo con el Dominante además de presentar una incomunicación con él (de acuerdo con los postulados de Lomnitz-Adler 1995).

La cultura íntima Dominante la he relacionado con personas que tienen apellidos españoles, anglosajones u otro, no-maya, y que tienen en su poder los medios de producción 
económicos, políticos y sociales: comerciantes, militares de alto rango y los trabajadores no manuales de compañías chicleras y madereras. Para los que integran la cultura íntima Residual, se les dividió en dos, Residual A y B. En términos generales al primero se le considera como el grueso de la población; ellos pueden tener cualquier apellido, y sus actividades económicas los involucran con elementos de producción de alimentos, y de trasformación, tales como trabajadores dedicados a actividades primarias - campesinos, chicleros, madereros, entre otros-, trabajadores manuales no calificados, así como a comerciantes al menudeo, como los que van de puerta en puerta. En cuanto al Residual B se le identificó por las madres que tenían características comparadas con el grupo A, pero que en un primer intento de clasificación caían como «in-asignables», dado que no había información de actividades laborales de padres y esposos o parejas, ellas iban a registrar a sus hijos solas, y no proporcionaron ninguna información acerca de ellas ni de sus familias ni esposos en el registro civil.

Por último, el grupo Emergente es aquel que surge y se renueva a través del tiempo, y que va teniendo enlaces de comunicación con el grupo Dominante, que se va mestizando («deculturando», utilizando los términos de Lomnitz-Adler 1995), recreando una nueva sociedad, la sociedad que ahora es la chetumaleña, la payobispense para Vallarta (2001). Es por tanto el grupo de profesionistas, empleados públicos, «la clase media», que se va construyendo con una identidad propia, además de que va ganando espacios de poder tanto económico como político.

Ubicadas las parejas, tanto por cohorte de nacimiento de la madre como al interior de una cultura íntima, se analizaron patrones y sistemas de familia, empleando en todos los análisis la comparación entre diferentes niveles geográficos, es decir, por toda la región, y a ambos lados de la frontera México-Honduras Británica (Distrito sur de Quintana Roo [DSQR] vs Distrito de Corozal) y así determinar comportamientos demográficos por grupos sociales. Para analizar patrones familiares por cultura íntima se evaluó el promedio en intervalos entre el nacimiento de la madre y el primer hijo, ${ }^{1}$ y el promedio de los intervalos 
intergenésicos, para conocer la existencia de sistemas familiares a partir del conocimiento del tamaño y tipo de las familias. En el primer rubro se obtuvo la Tasa Global de Fecundidad (TGF), por periodo y por cohorte. Para el segundo rubro se establecieron tipos de familias a partir de si eran endógamas o exógamas, indicador importante para analizar elementos de asimilación estructural (Alba y Golden 1986, Gans 1999).

Con estos datos será posible presentar una serie de hipótesis sobre decisiones de formación familiar, y a su vez comportamientos demográficos, en los cuales las parejas operan, matizadas estas decisiones por su ubicación en la escala social de etnia y clase — cultura íntima-, por la historia migratoria de las parejas, y a procesos de asimilación de las parejas al cruzar las fronteras nacionales.

\section{RESULTADOS}

ESPACIAMIENTO ENTRE NACIMIENTOS

El intervalo entre el nacimiento de la madre a la fecha del nacimiento del primogénito en el Distrito del sur de Quintana Roo es a una edad más tardía, 2.5 años después, del que se presenta entre las mujeres del Distrito de Corozal (20.3 años vs. 17.8 años) (Cuadro 2).

Cuando se analiza este comportamiento por cultura íntima al interior de cada Distrito, se observa que en el DSQR la cultura íntima Dominante es la que tiene más tardíamente el nacimiento del primogénito, a los 22.6 años, más de dos años y medio que de las madres del Distrito en general; mientras, las mujeres ubicadas en la cultura íntima Emergente y Residual A se apegan bastante al patrón general, con ligeros atrasos para la Emergente ( 0.5 años más) o adelantos, como se ve en la Residual A (0.2 años antes que el patrón general). Las mujeres ubicadas en la Residual B, los tienen a una edad promedio de 15.5 años, siendo las más jóvenes para todo la región, si se les compara tanto al interior del DSQR como con mujeres de Corozal ubicadas igualmente dentro de esta cultura íntima, ya que ellas los tienen a los 18 años en promedio -2.5 años después que las del DSQR (Cuadro 2). 
Ya en el mismo intervalo pero del Distrito de Corozal, tanto el patrón general como al interior de cada cultura íntima es muy similar, sobre todo el Residual A, en comparación con todo el Distrito - ambos 17.8 años-, pues el conjunto está alrededor de los 18 años, siendo las mujeres de la cultura íntima Emergente las que tienen sus hijos de manera más temprana, 17.5 años, y las que las tienen más tardíamente son de la Residual B, 18 años (Cuadro 2).

Para los intervalos intergenésicos se observa que hay poca diferencia entre ambos Distritos; sin embargo, estos son menores entre las madres del DSQR con respecto a la madres del Distrito de Corozal. Cuando se les divide por culturas íntimas el comportamiento es diferente, pues para las madres de la cultura íntima Emergente son más largos en el DSQR, entre 0.1 años y 5.8 , que los de su contraparte de Corozal, 0.1 y 0.5 . Para las madres de la cultura íntima Residual A cambia una vez más la situación y se apega al comportamiento general de los Distritos, es decir, las madres de la Residual A del DSQR tienen intervalos más cortos que las de Corozal. Para terminar, las madres de la cultura íntima Residual B del DSQR tienen a sus hijos con mayor espacio entre el primer y segundo hijo, 1.5 años más que las de Corozal.

En términos generales, las mujeres reconstruidas están dejando un espacio entre cada nacimiento, entre los 2.3 y 3 años, teniendo ligeramente más espaciado los nacimientos en las madres del DSQR. Esto puede ser un dato ficticio, pues si analizamos las medianas en lugar de los promedios, vemos que a ambos lados de la frontera estas mujeres reconstruidas que espacian el nacimiento de sus hijos en forma similar (2.5 años), y solamente las Residuales A y B son mayores en el Distrito de Corozal en comparación con las del DSQR, y para las de la Emergente en el DSQR son ligeramente más espaciados sus nacimientos, cuando se analizan sus medianas (Cuadro 2). 
del siglo XX. Análisis socio-demográfico del impacto de

la migración en la formación familiar

Cuadro 2. Edad promedio en años de la fecha de nacimiento de la madre* al primer hijo, y promedio de años de los intervalos inter-genésicos de las mujeres seleccionadas por Región, Distrito y Cultura íntima. Estadísticas vitales 1885-1955.

\begin{tabular}{|c|c|c|c|c|c|c|c|c|c|}
\hline \multirow{3}{*}{ Intervalos } & \multicolumn{9}{|c|}{ Promedio en años en los intervalos inter-genésicos de las mujeres seleccionadas } \\
\hline & \multicolumn{5}{|c|}{ Distrito sur de Quintana Roo } & \multicolumn{4}{|c|}{ Distrito de Corozal** } \\
\hline & Distrito & Dominante & Emergente & Residual A & Residual B & Distrito & Emergente & Residual A & Residual B \\
\hline $\begin{array}{l}\text { Fecha de nacimiento de } \\
\text { la madre al primer hijo }\end{array}$ & 20.3 & 22.6 & 20.8 & 20.1 & 15.5 & 17.8 & 17.5 & 17.8 & 18.0 \\
\hline Del primero a segundo hijo & 3.2 & 3.8 & 3.3 & 3.1 & 4.2 & 2.8 & 2.2 & 2.8 & 2.7 \\
\hline Segundo al tercer hijo & 2.6 & & 2.5 & 2.6 & & 2.7 & 2.4 & 2.8 & 2.6 \\
\hline Tercer al cuarto & 2.6 & & 3.2 & 2.4 & & 2.7 & 2.7 & 2.8 & 2.5 \\
\hline Cuarto al quinto & 2.4 & & 2.5 & 2.3 & & 2.4 & 1.6 & 2.4 & 5.2 \\
\hline Quinto al sexto & 2.4 & & 2.4 & 2.5 & & 2.8 & 2.2 & 2.8 & 3.0 \\
\hline Sexto al séptimo & 2.8 & & 2.8 & 2.8 & & 2.5 & 2.2 & 2.5 & 3.5 \\
\hline Séptimo al octavo & 1.7 & & 1.9 & 1.6 & & 2.7 & 2.8 & 2.7 & 3.8 \\
\hline Octavo al noveno & 2.0 & & 2.0 & 1.9 & & 2.5 & 2.1 & 2.6 & 1.3 \\
\hline Noveno al décimo & 2.1 & & 1.2 & 2.6 & & 2.5 & 2.6 & 2.6 & 1.8 \\
\hline Décimo al onceavo & 4.8 & & 8.2 & 1.4 & & 2.2 & 2.4 & 2.2 & \\
\hline Onceavo al doceavo & & & & & & 1.9 & 3.1 & 1.7 & \\
\hline \begin{tabular}{|l|} 
Doceavo al treceavo \\
\end{tabular} & & & & & & 2.2 & 1.5 & 2.4 & \\
\hline Treceavo al catorceavo & & & & & & 2.4 & 2.4 & & \\
\hline Promedio de los intervalos & 2.7 & & 3.0 & 2.3 & & 2.6 & 2.3 & 2.6 & 2.9 \\
\hline Mediana & 2.5 & & 2.5 & 2.5 & & 2.5 & 2.4 & 2.6 & 2.7 \\
\hline
\end{tabular}

*Universo muestral $=415$ madres seleccionadas.

**No hay personas asignadas a la cultura íntima Dominante en el Distrito de Corozal.

Fuente: Cálculos propios. Estadísticas vitales, 1885-1955 


\section{LA FECUNDIDAD EN LA REGIÓN DE ESTUDIO}

Utilizando indicadores más finos sobre el comportamiento de la fecundidad de estas mujeres que han sido seleccionadas al azar, se obtuvieron las Tasas Globales de Fecundidad (TGF) por periodo. En el Cuadro 3 se presentan las tasas por periodo para los dos Distritos analizados. De 1900 a 1909 se observa que las mujeres que representan al Distrito de Corozal tienen mayores TGF que las mujeres del DSQR. Esta es la época de la llegada de los primeros migrantes a las localidades formadas por ellos, que antes eran y aún lo seguían siendo campamentos madereros y chicleros. Las primeras llegan a tener hasta 6.37 y 4.81 hijos por mujer, para los quinquenios 1900-1904 y 1905-1909, mientras que las del sur de Quintana Roo no llegan ni al nivel de remplazo (2.1 hijos por mujer), ya que las tasas son 0.25 y 0.72 hijos por mujer, para los quinquenios señalados. Esto nos indica que las mujeres de Corozal posiblemente mantenían una fecundidad natural constante a lo largo del tiempo, mientras que las del sur de Quintana Roo pudieron haber retrasado muchos de sus embarazos y obviamente por ello sus nacimientos, debido al proceso migratorio que sufrieron, al haberse asentado en nuevos lugares teniendo que adaptarse a nuevas condiciones de vida.

Sin embargo hay un cambio cuantitativo en el comportamiento de la fecundidad de las mujeres que habitaron tanto el sur de Quintana Roo como de Corozal a principios del siglo XX. La TGF empieza a incrementarse conforme van pasando los años, pues de 0.25 hijos por mujer éstas llegan a elevarse hasta 3.55 hijos por mujer en 1910-1914; posterior a ello su comportamiento es más errático, pues baja en los siguientes quinquenios hasta 1.32 hijos en 1920-1924, para que a partir de este punto vuelva a incrementarse en 1930-1934 hasta 3.15 hijos por mujer (Cuadro 3). 
Cuadro 3. Tasa Global de Fecundidad" por periodo de los Distritos y por Cultura íntima.

\begin{tabular}{|c|c|c|c|c|c|c|c|c|c|c|}
\hline \multirow{3}{*}{ Periodos } & \multicolumn{10}{|c|}{ Tasa Global de Fecundidad por periodos de las mujeres seleccionadas } \\
\hline & \multicolumn{2}{|c|}{ Distrito } & \multicolumn{2}{|c|}{ Dominante } & \multicolumn{2}{|c|}{ Emergente } & \multicolumn{2}{|c|}{ Residual A } & \multicolumn{2}{|c|}{ Residual B } \\
\hline & DSQR & \begin{tabular}{|c|} 
D. \\
Corozal \\
\end{tabular} & DSQR & $\begin{array}{c}\text { D. } \\
\text { Corozal } \\
\end{array}$ & DSQR & \begin{tabular}{|c|} 
D. \\
Corozal \\
\end{tabular} & DSQR & \begin{tabular}{|c|} 
D. \\
Corozal \\
\end{tabular} & DSQR & \begin{tabular}{|c|} 
D. \\
Corozal \\
\end{tabular} \\
\hline $1900-1904$ & 0.25 & 6.37 & 0.00 & 0.00 & 0.00 & 0.20 & 0.30 & 5.26 & 0.00 & 0.10 \\
\hline 1905-1909 & 0.72 & 4.81 & 0.00 & 0.00 & 0.40 & 0.40 & 0.50 & 4.50 & 0.00 & 0.20 \\
\hline $1910-1914$ & 3.55 & 3.14 & 0.80 & 0.20 & 2.90 & 0.40 & 2.95 & 3.06 & 0.00 & 0.80 \\
\hline 1915-1919 & 2.83 & 1.15 & 0.40 & 0.40 & 2.70 & 0.60 & 2.61 & 1.01 & 0.20 & 0.00 \\
\hline 1920-1924 & 1.32 & 0.89 & 0.00 & 0.20 & 1.73 & 0.00 & 0.96 & 0.90 & 0.00 & 0.00 \\
\hline 1925-1929 & 2.64 & 0.24 & 1.00 & 0.00 & 2.33 & 0.40 & 2.22 & 0.21 & 0.00 & 0.00 \\
\hline 1930-1934 & 3.15 & & 0.20 & & 2.56 & & 3.26 & & 0.20 & \\
\hline
\end{tabular}

*Universo muestral $=415$ madres seleccionadas.

Fuente: Cálculos propios. Estadísticas vitales, 1885-1955.

Sucede lo contrario en el Distrito de Corozal, pues si bien presentaban elevadas tasas de fecundidad en los dos primeros quinquenios del siglo XX, más allá del remplazo, este comportamiento se fue yendo hacia la baja de manera continua, siendo que a partir de 1915-1919 ya se encontraban por abajo del nivel de remplazo, hasta llegar en 1925-1929 a los 0.24 hijos por mujer (Cuadro 3). La baja en la fecundidad para estos periodos tanto para el sur de Quintana Roo como para Corozal, se pudieron deber a las fluctuaciones de crisis económicas mundiales (Kuntz 2007) o bien a efectos de crisis políticas internas y de abandono del territorio por parte del gobierno, sobre todo para Quintana Roo (Aguirre et al. 1925, Macías Richard 1997b).

Este comportamiento es diferente cuando se divide a las mujeres por culturas íntimas. Para la cultura íntima Dominante se logró estimar las TGF en los quinquenios 1910-1914 y 1915- 
1919, siendo los dos únicos quinquenios en que se puede hacer una comparación entre ambos Distritos (en 1920-1924 sólo hay información para Corozal, y en 1925-29 y 1930-34 sólo para DSQR). Si bien en 1910-1914 las mujeres de la cultura íntima Dominante de Quintana Roo tienen una tasa más elevada, al siguiente quinquenio ésta baja, poniéndose a la par con la de Corozal, la cual se eleva ligeramente del quinquenio anterior a 0.4 hijos por mujer (Cuadro 3).

El comportamiento de la fecundidad, a partir de TGF de la cultura íntima Residual A, se pudo analizar para todos los periodos señalados en ambos Distritos, haciendo más rica la comparación al interior de este grupo. El grupo Residual A del Distrito de Corozal presenta en los tres primeros quinquenios mayores TGF que las mujeres del mismo grupo de Quintana Roo (5.26, 4.5 y 3.06 hijos por mujer), y todas ellas están por arriba del nivel de reemplazo. A partir del quinquenio 1915-1919, las mujeres del DSQR comienzan a tener mayores TGF que las de Corozal; para el quinquenio 1920-1924 se encuentran abajo del reemplazo. Este nivel, por arriba del reemplazo, ya lo habían logrado desde el quinquenio 1910-1914 a pesar de que era más bajo que el de Corozal (Cuadro 3). En general, el comportamiento de la fecundidad para este grupo es el reflejo del comportamiento general de los distritos que se describieron al principio, es decir, presenta la mayor TGF que luego disminuye de forma gradual; para las mujeres del grupo Residual A del DSQR tienen exactamente el mismo comportamiento de todo el Distrito al que pertenecen pero con tasas ligeramente más bajas (Cuadro 3).

Para la cultura íntima Emergente hay un comportamiento ligeramente diferente, sobre todo para las mujeres del DSQR, ya que refleja el comportamiento general de todo el Distrito (al igual que el Residual A del mismo Distrito) pero con tasas ligeramente más bajas que las generales, pero más altas que las del Residual A sobre todo en los quinquenios 1915-1919 hasta 1925-1929. El nivel de remplazo lo sostienen en todos los quinquenios desde 19101914 hasta 1930-1934 (excepción 1920-1924). En cuanto al Distrito de Corozal en esta cultura íntima en ninguno de los quinquenios que representan las tres primeras década del 
siglo XX llegan al nivel de reemplazo y, por ende, con menores tasas que las del DSQR que es en el único grupo en que se ve este comportamiento (Cuadro 3).

Por último, el grupo Residual B tiene muy pocas parejas e hijos registrados para el DSQR, por lo que sus tasas son muy bajas y sólo registradas en los quinquenios 1915-1919 y 19301934 (0.2 hijos por mujer en ambos quinquenios). Por estas razones la comparación del comportamiento de la fecundidad sólo se hace al interior de la misma cultura íntima Residual B a lo largo del tiempo, de la cual se tiene registrada desde los primeros 15 años del siglo XX. De tal manera, la TGF a lo largo de los tres quinquenios se va elevando de manera paulatina (0.1, 0.2 y 0.8 hijos por mujer), pero sin llegar nunca al reemplazo, es decir, 2 hijos por mujer (Cuadro 3).

Ahora bien, si se analiza la fecundidad por cohorte se puede comprender la intensidad y el calendario de la fecundidad. En la Figura 1 se presentan las generaciones 1889 a 1923 divididas por grupos quinquenales pertenecientes al DSQR. Se observa que el calendario de estas mujeres es «ligeramente» homogéneo, presentando el inicio de una etapa reproductiva muy joven, 10-14 años de edad, sobre todo en las generaciones 1894-1898, y de la generación 1904-1908 hasta la generación 1929-1933, aunque la tasa es relativamente más baja que las tasas del mismo grupo de edad de las generaciones de Corozal (0.31 vs. 0.04, 1894-1898; 0.03 vs. 0.00, 1899-1903; 0.26 vs. $0.11,1904-1908 ; 0.27$ vs. 0.08, 1909-1913 y, 0.2 vs. 0.13, 1914-1918).

La intensidad de la fecundidad entre las cohortes se observa que va aumentando, a excepción del comportamiento errático de la generación 1889-1893, que su fecundidad se encuentra por encima en las edades 20-24 y 25-29 de las generaciones $1899-1903$ y 19041908. Otro comportamiento notoriamente errático es el de la generación 1894-1898, pues mientras que en casi todas las generaciones - la excepción es la generación 1904-1908 donde el pico en la fecundidad está en el grupo 20-24 años (0.94 hijos por mujer, mientras que 0.86 hijos para 25-29 años) — la fecundidad más alta está en el grupo 25-29 años, en 
esta generación se presentó uno de los tres puntos más bajos de fecundidad. Esto significa, quizá, la existencia de un subregistro de los nacimientos ocurridos para esta generación entre los años 1919-1927.

Figura 1. Tasas Específicas de Fecundidad por edades quinquenales por generación de la madre del Distrito sur de Quintana Roo.

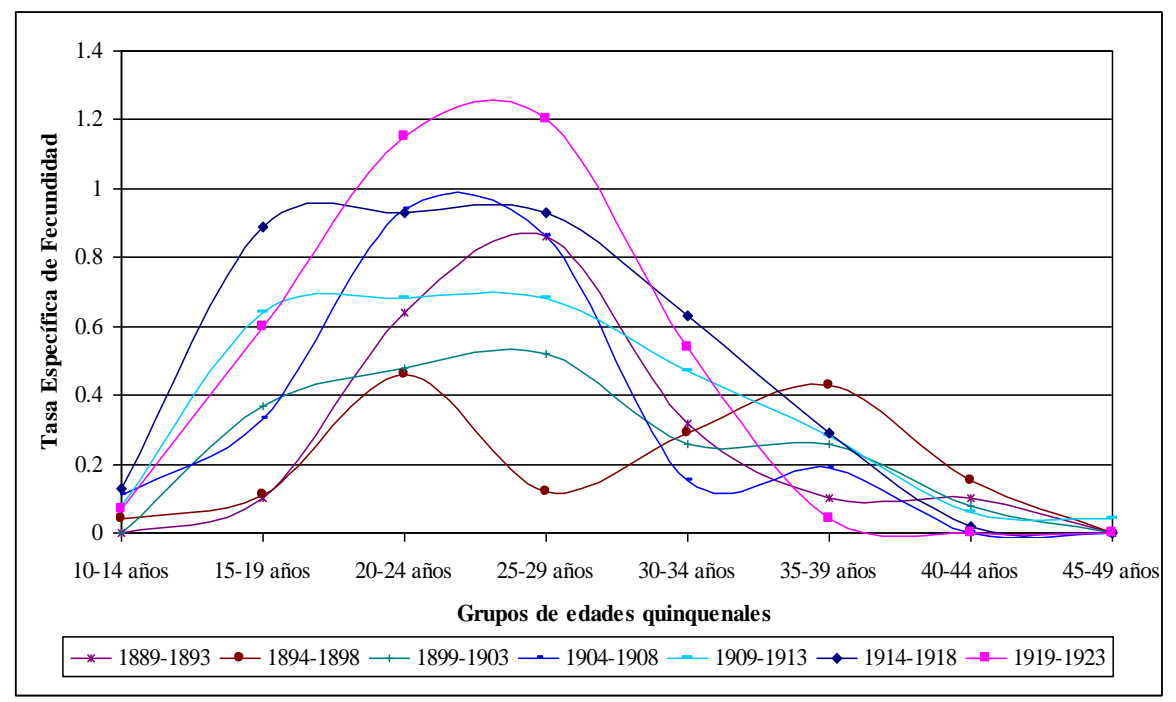

Fuente: Cálculos propios. Estadísticas vitales 1885-1955.

Tomando en cuenta a las generaciones del sur de Quintana Roo que tienen «historias reproductivas completas», cohortes de 1889-1893 a 1899-1903 —que corresponde a los nacimientos ocurridos entre 1899 a 1952 - , se observa su más alta intensidad en los grupos de edad de 25 a 29 años, y a partir de estas edades la intensidad va bajando gradualmente. En cuanto al Distrito de Corozal el panorama es otro, ya que se cuenta con «historias reproductivas completas» para las generaciones de 1864-1868 —nacimientos de 18741917 - a 1879-1883 — nacimientos de 1889-1932 — con relativamente buena información. De manera general, y en comparación con el comportamiento de la fecundidad, ocurre un rejuvenecimiento en el calendario de la misma, pues en las generaciones 1864-68, 1869-73, 
$1874-78,1894-98$ y $1899-1903$, esta ocurre a mayor intensidad en el grupo de edad de 20 a 24 años, mientras que en las generaciones $1879-83$ y $1884-88$ el comportamiento es errático, retrasándose la intensidad mayor al siguiente grupo quinquenal de edad (Figura 2).

Figura 2. Tasas Específicas de Fecundidad por edades quinquenales por generación de la madre del Distrito de Corozal.

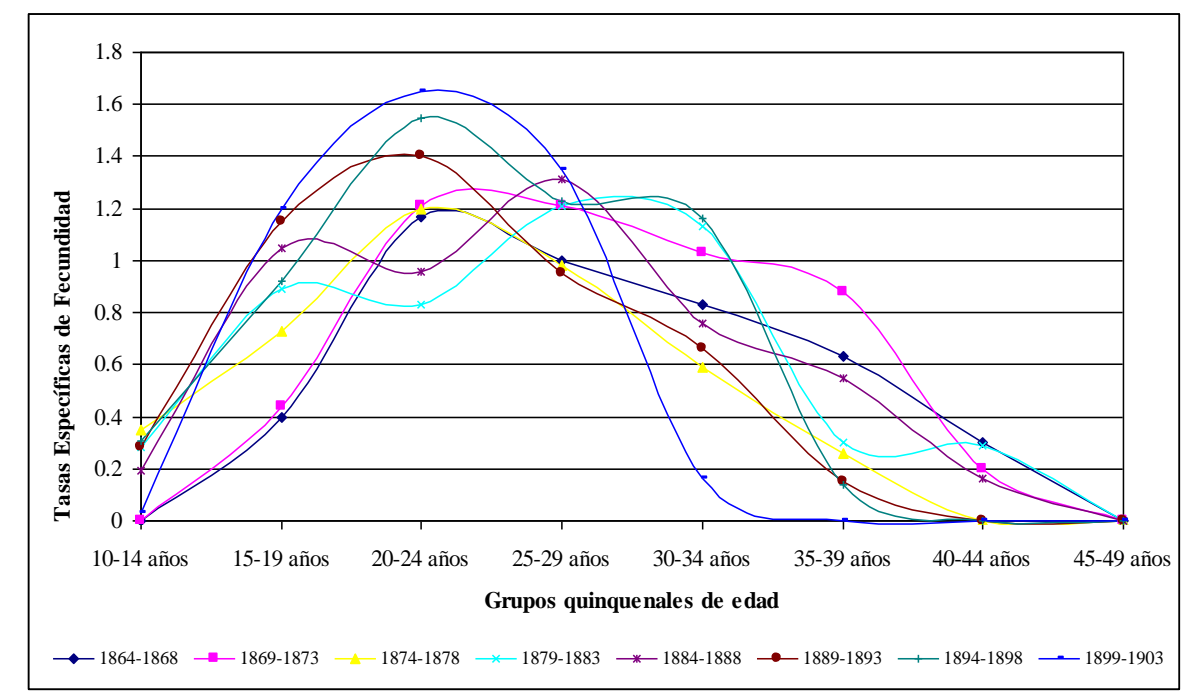

Fuente: Cálculos propios. Estadísticas vitales 1885-1955.

Como sucedió con el DSQR, la fecundidad es precoz, pues comienza a edad muy temprana, alrededor de 10 años, pero lo que tienen las mujeres de esta muestra de Corozal es que su fecundidad es más elevada que la de las mujeres del otro Distrito (Figuras 1 y 2) y, al igual que en el sur de Quintana, se va elevando al seguir las generaciones en el tiempo.

En cuanto a las Tasas Globales de Fecundidad (TGF) por cohorte de las madres se observan tendencias de «sube y baja» en la fecundidad global por mujer a lo largo de las cohortes. Por ejemplo, para las mujeres seleccionadas del sur de Quintana Roo hay dos tendencias a la alza de fecundidad, una para las cohortes que van de 1869-73 a 1884-1888, con una tasa 
de 0.4 hijos por mujer a 2.91 hijos por mujer — nacimientos de 1879 a 1937 - y la otra de 1894-1898 a 1914-1918, con 1.59 hijos por mujer a 3.82 hijos por mujer —nacimientos 1904 a 1954, para el grupo de edad 40-44 años en este último año (Figura 3).

Según la tendencia a la baja la vemos en las cohortes 1884-1888 a 1894-1898 —nacimientos de 1894 a 1947-y de 1914-1918 a 1929-1933 —nacimientos de 1924 a 1953 para el grupo de edad de 20-24 años- que van, en el primer caso, y una vez que alcanza la máxima TGF previa, de 2.91 hijos a 1.59 hijos por mujer; y en el segundo grupo de cohortes, 3.82 hijos a 2.91 hijos por mujer. En cuanto a la segunda reducción de la fecundidad en el grupo de cohortes es más bien ficticia, ya que falta por registrar a más de la mitad de los hijos nacidos vivos que van de los años 1956 a 1982, sobre todo para las generaciones de las madres de 1916 a 1933, por ende, esto hace que la fecundidad se vea reducida (Figura 3).

Por tanto, se puede considerar que, dejando de lado esa segunda tendencia a la baja de la fecundidad, esta se mantiene a la alza conforme pasan las generaciones en las mujeres reconstruidas del DSQR, acorde con lo visto en la Figura 1, a lo que cabría preguntarse qué fenómeno ocurrió para que la fecundidad se viera disminuida en las generaciones 18841888 a $1894-1898$.

En cuanto al registro de la TGF para las mujeres reconstruidas del Distrito de Corozal se observan tres etapas de alza de la fecundidad al interior del mismo. Uno de ellos es 1859-63 a 1869-73 (nacimientos de 1869 a 1922) con una TGF de 3.0 a 4.97 hijos por mujer; de 1874-78 a 1884-1888 (nacimientos 1884 a 1933, en grupos de edad de 45 a 49 años, faltando por registrar cuatro años para tener la historia reproductiva completa), con tasas de 4.12 a 4.98 hijos por mujer, y de 1889-1893 a 1894-1898 (nacimientos 1899 a 1933 en el grupo de edad de 35 a 39 años) con 4.59 a 5.31 hijos por mujer (Figura 3). 
Figura 3. Tasas Globales de Fecundidad por generación de la madre del Distrito del sur de Quintana Roo y del Distrito de Corozal.

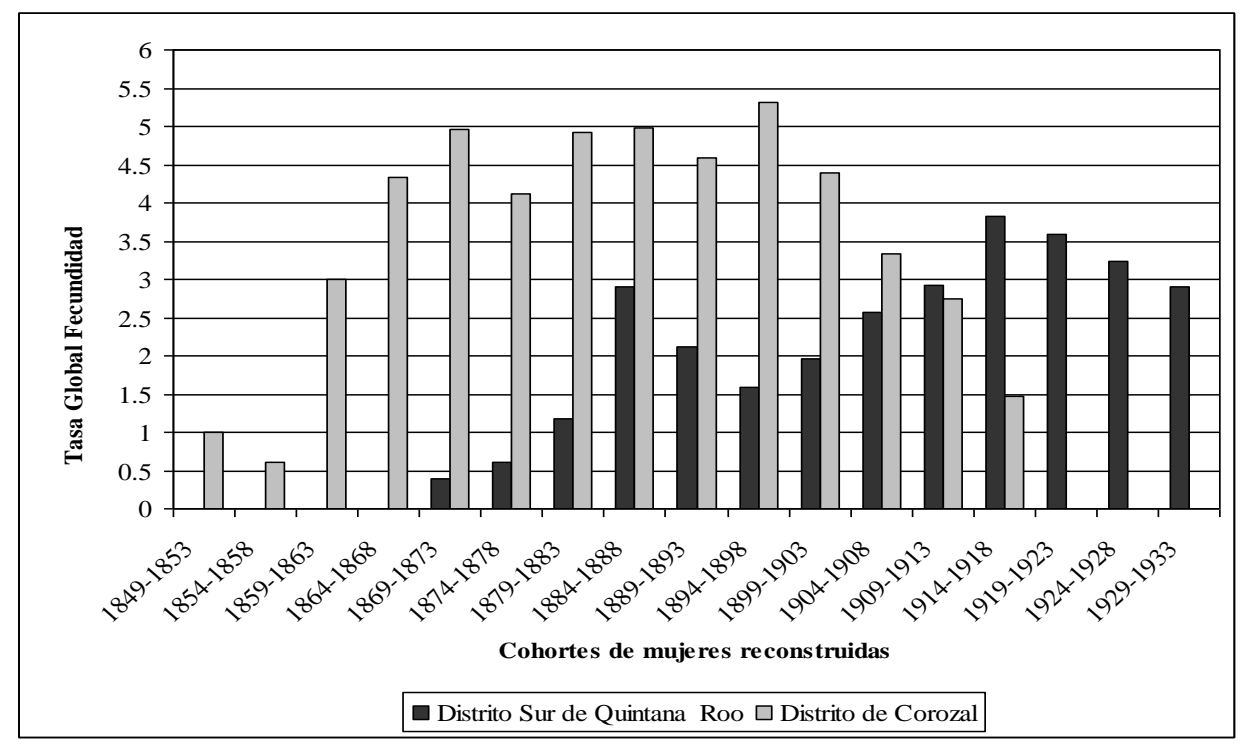

Fuente: Cálculos propios. Estadísticas vitales 1885-1955.

De esta última generación 1894-1898, la TGF por cohorte se ve disminuida paulatinamente yendo de su nivel máximo que alcanzan la TGF por cohorte que es de 5.31 hijos por mujer y de allí hasta los 1.47 hijos por mujer en la generación 1914-1918 (nacimientos 1904 a 1933, para el grupo de edad 15 a 19 años de edad para la generación de 1918) [Figura 3]. Esta disminución, como la ocurrida para el DSQR, es ficticia, ya que no se registran todos los nacimientos ocurridos para el Distrito pues la fuente de datos consultada llega hasta 1933.

Comparando a ambos Distritos por la TGF por cohorte, el Distrito de Quintana Roo presenta TGF menores en todas las generaciones, lo cual puede estar asociado al efecto de la migración en ellas al tener una menor descendencia que las mujeres de Corozal a lo largo de las generaciones de la mujeres, y esta fecundidad solo va aumentando a lo largo de cada 
generación conforme a las localidades, y las condiciones de vida en estas permite sostener y mantener mayores niveles de fecundidad.

\section{TIPO DE FAMILIAS: PAREJAS ENDO/EXOGÁMICAS}

De las 864 parejas reconstruidas, en $42.2 \%$ (365 casos) se logró determinar el lugar de origen de los padres de los hijos que nacieron y se registraron en la región de estudio. Para entender mejor esta distribución se dividió a la muestra por Distritos de donde se inscribió el nacimiento, DSQR y Distrito de Corozal (Cuadro 4). En el DSQR se aprecia un porcentaje mayor de exogamia hacia fuera de la región, es decir, ambos padres no son de la región de estudio, $35.4 \%$, y lo que se podría decir como endogamia, donde ambos padres son de Quintana Roo ocupa el segundo lugar más bajo con 3.4\% de los casos. Siguiendo con este patrón exogámico se observa que hay en el DSQR padres y madres nativos de Corozal, pero que su pareja no es de la región (11.9\% y 12.9\%, respectivamente) e igualmente el padre y la madre sí son de Quintana, pero sus parejas no son de la región (12.2\% y 7.5\%). Por último, es de notar que los padres y madres que tienen parejas originarias de Corozal, así como ambos padres, son de este Distrito y ocupan los menores porcentajes de la muestra (3.7\%, $1.4 \%$ y $3.4 \%$, respectivamente).

Ahora bien, si se observa al interior de las culturas íntimas, en todos los grupos la categoría «ambos padres no son nativos de la región» tiene el mayor porcentaje (1.7\% en la Dominante, $8.5 \%$ Emergente, 24.8\% Residual A y 0.3\% Residual B) de toda la muestra (Cuadro 4). Las diferencias se encuentran al interior de las demás categorías para cada una de las culturas íntimas. 
Cuadro 4. Endo/exogamia según origen de padres y madres, Distrito sur de Quintana Roo y

Distrito de Corozal, por culturas íntimas.

\begin{tabular}{|c|c|c|c|c|c|c|}
\hline & \multirow{2}{*}{$\begin{array}{l}\text { Lugares de origen de padres } \\
\text { y madres de toda la región }\end{array}$} & \multicolumn{4}{|c|}{ Culturas íntimas } & \multirow[b]{2}{*}{ Total } \\
\hline & & Dominante & Emergente & $\begin{array}{c}\text { Residual } \\
\text { A }\end{array}$ & $\begin{array}{c}\text { Residual } \\
\text { B }\end{array}$ & \\
\hline \multirow{10}{*}{ 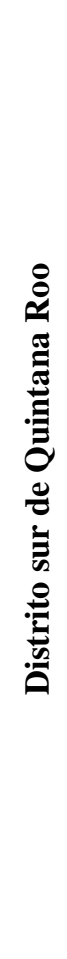 } & $\begin{array}{l}\text { Ambos padres no son nativos de } \\
\text { Corozal ni de Quintana Roo }\end{array}$ & $1.7 \%$ & $8.5 \%$ & $24.8 \%$ & $0.3 \%$ & $35.4 \%$ \\
\hline & $\begin{array}{l}\text { Ambos padres son nativos de } \\
\text { Quintana Roo } \\
\end{array}$ & & $1.0 \%$ & $2.4 \%$ & & $3.4 \%$ \\
\hline & $\begin{array}{l}\text { Ambos padres son nativos de } \\
\text { Corozal }\end{array}$ & & $2.0 \%$ & $9.5 \%$ & & $11.6 \%$ \\
\hline & $\begin{array}{l}\text { Padre es nativo de Corozal y } \\
\text { madre no es nativa de Corozal ni } \\
\text { de Quintana Roo }\end{array}$ & & $5.1 \%$ & $6.8 \%$ & & $11.9 \%$ \\
\hline & $\begin{array}{l}\text { Madre es nativa de Corozal y } \\
\text { padre no es nativo de Corozal ni } \\
\text { de Quintana Roo }\end{array}$ & $0.3 \%$ & $1.0 \%$ & $11.6 \%$ & & $12.9 \%$ \\
\hline & $\begin{array}{l}\text { Padre es nativo de Quintana Roo } \\
\text { y madre no es nativa de Corozal } \\
\text { ni de Quintana Roo }\end{array}$ & & $3.7 \%$ & $8.5 \%$ & & $12.2 \%$ \\
\hline & $\begin{array}{l}\text { Madre es nativa de Quintana Roo } \\
\text { y padre no es nativo de Corozal ni } \\
\text { de Quintana Roo } \\
\end{array}$ & & $1.0 \%$ & $6.5 \%$ & & $7.5 \%$ \\
\hline & $\begin{array}{l}\text { Padre es nativo de Quintana Roo } \\
\text { y madre es nativa de Corozal }\end{array}$ & & $0.7 \%$ & $3.1 \%$ & & $3.7 \%$ \\
\hline & $\begin{array}{l}\text { Madre es nativa de Quintana Roo } \\
\text { y padre es nativo de Corozal } \\
\end{array}$ & & $0.7 \%$ & $0.7 \%$ & & $1.4 \%$ \\
\hline & Total & $2.0 \%$ & $23.8 \%$ & $73.8 \%$ & $0.3 \%$ & $100 \%(294)$ \\
\hline \multirow{6}{*}{ 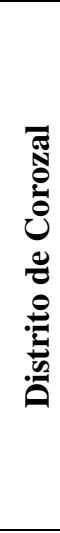 } & $\begin{array}{l}\text { Ambos padres no son nativos de } \\
\text { Corozal ni de Quintana Roo }\end{array}$ & & $1.4 \%$ & $12.7 \%$ & & $14.1 \%$ \\
\hline & $\begin{array}{l}\text { Ambos padres son nativos de } \\
\text { Corozal }\end{array}$ & & $7.0 \%$ & $43.7 \%$ & & $50.7 \%$ \\
\hline & $\begin{array}{l}\text { Padre es nativo de Corozal y } \\
\text { madre no es nativa de Corozal ni } \\
\text { de Quintana Roo }\end{array}$ & & $1.4 \%$ & $19.7 \%$ & & $21.1 \%$ \\
\hline & $\begin{array}{l}\text { Madre es nativa de Corozal y } \\
\text { padre no es nativo de Corozal ni } \\
\text { de Quintana Roo } \\
\end{array}$ & & $1.4 \%$ & $11.3 \%$ & & $12.7 \%$ \\
\hline & $\begin{array}{l}\text { Madre es nativa de Quintana Roo } \\
\text { y padre es nativo de Corozal }\end{array}$ & & $0.0 \%$ & $1.4 \%$ & & $1.4 \%$ \\
\hline & Total & & $11.3 \%$ & $88.7 \%$ & & $100 \%(71)$ \\
\hline
\end{tabular}

Nota: el valor absoluto total se ofrece en la última columna, última línea, entre paréntesis. Con él se puede realizar fácilmente el cálculo del valor absoluto al interior de cada casilla.

Fuente: Cálculos propios. Estadísticas vitales, 1885-1955. 
De tal manera, en la cultura íntima Dominante hay una madre que es nativa de Corozal y su pareja era fuera de la región, representando $0.3 \%$ de la muestra (Cuadro 4). Para la cultura íntima Emergente hay parejas en todas las categorías, pero las más representadas son los padres nativos de Corozal y de Quintana Roo, y la madre no es de la región de estudio (5.1\% y $3.7 \%$, respectivamente), así como la categoría «ambos padres son nativos de Corozal» con $2 \%$ de toda la muestra (Cuadro 4).

La cultura íntima Residual A es la que tiene el mayor porcentaje de las parejas de toda la muestra con respecto a las otras culturas íntimas (73.8\% de toda la muestra). Esta cultura íntima se diferencia de la cultura Emergente, pues de igual manera tiene representación de parejas en todas las categorías, en «madre es nativa de Corozal y el padre no es de la región» representan $11.6 \%$, le sigue en la mayor representación «ambos padres son de Corozal» (9.5\%), y el padre es nativo de Quintana Roo y la madre no es de la región con $8.5 \%$ (Cuadro 4).

Por último, la cultura íntima Residual B, como era de esperarse dada la baja cantidad de parejas que hay para este grupo dentro de las parejas que registraron hijos dentro de Quintana Roo, solamente hay una pareja en la cual ambos padres no son nativos de la región, denotando únicamente $0.3 \%$ de la muestra total (Cuadro 4).

Es de notar la baja proporción que tiene la categoría «ambos padres son nativos de Quintana Roo» al interior de todas las culturas íntimas (0\% en la Dominante, $1 \%$ Emergente, 2.4\% Residual A y 0\% Residual B), denotando así una baja proporción de endogamia para los nativos de Quintana Roo (Cuadro 4).

Para el Distrito de Corozal el comportamiento es diferente, además de que las categorías se reducen a cinco, mientras que para el sur de Quintana Roo fueron ocho categorías registradas. De manera global, se observa en el Cuadro 4 que 50.7\% de la muestra fueron «ambos padres son nativos de Corozal» (para el sur de Quintana Roo, como se mencionó 
para la categoría «ambos padres son nativos de Quintana Roo», es de tan sólo 3.4\%). Le sigue en importancia «el padre es nativo de Corozal, pero la madre no es de la región» con 21.1\%, y por último en importancia: «ambos padres no son nativos de la región» (14.1\%), la madre es nativa de Corozal y el padre no es de la región (12.7\%). Es de notar que no hay padres varones nativos de Quintana Roo, y solamente hay una que es nativa de Quintana Roo y el padre es de Corozal con $1.4 \%$.

Al interior de las culturas íntimas para el Distrito de Corozal, solamente se tiene información de los orígenes de las parejas en las culturas íntimas Emergente y Residual A. Es de notar que para Residual B no hay datos disponibles debido a que, y a pesar de que su mayor contingente es en este Distrito de Corozal, no se tiene el lugar de origen de la madre, como también son madres que registran a sus hijos sin su pareja.

Para la cultura íntima Emergente, el mayor porcentaje se encuentra en la categoría «ambos padres son nativos de Corozal» con $7 \%$ de la muestra total, mientras que las categorías «ambos padres no son de la región», el padre y la madre son nativos de Corozal, y sus correspondientes parejas no son nativas de la región, representan $1.4 \%$ en todas las categorías (Cuadro 4).

Para la cultura íntima Residual A, al igual que la cultura íntima Emergente, el mayor porcentaje se encuentra en la categoría «ambos padres son nativos de Corozal» (43.7\%), siguiendo el padre es nativo de Corozal y la madre no es la región con 19.7\% y «ambos padres no son nativos de la región» (12.7\%), el último de importancia es el de la madre es nativa de Corozal y el padre no es de la región con $11.3 \%$ de la muestra. En esta cultura íntima es donde está la única pareja que la madre es nativa de Quintana Roo y el padre es de Corozal, de la cual ya se había hecho mención en párrafos anteriores (Cuadro 4). 
La hipótesis de trabajo que se planteó fue que el comportamiento de endo/exogamia iría cambiando conforme pasaba el tiempo, sobre todo para Quintana Roo, por un proceso de asimilación. Por consiguiente en los cuadros 5 y 6 se expone el decenio de nacimiento del primogénito registrado, tanto para los registros ubicados dentro del Distrito sur de Quintana Roo como del Distrito de Corozal ordenados en cuatro categorías: Padres exógamos, padres endógamos nativos de Quintana Roo, padres endógamos nativos de Corozal, y la suma de estos dos en una sola categoría, padres endógamos.

Cuadro 5. Cambio de endo/exogamia de los padres a través del tiempo de generación de nacimiento del hijo registrado en el Distrito sur de Quintana Roo.

\begin{tabular}{|l|r|r|r|r|r|}
\hline $\begin{array}{l}\text { Decenio de } \\
\text { nacimiento del } \\
\text { hijo registrado }\end{array}$ & $\begin{array}{r}\text { Padres } \\
\text { exógamos }\end{array}$ & $\begin{array}{c}\text { Padres endógamos } \\
\text { de Quintana Roo }\end{array}$ & $\begin{array}{c}\text { Padres endógamos } \\
\text { de Corozal }\end{array}$ & $\begin{array}{c}\text { Padres } \\
\text { endógamos }\end{array}$ & Total \\
\hline $1890-1899$ & $0.68 \%$ & & & & $0.68 \%$ \\
\hline $1900-1910$ & $3.07 \%$ & & $2.05 \%$ & $2.05 \%$ & $5.12 \%$ \\
\hline $1910-1919$ & $9.90 \%$ & $0.34 \%$ & $2.05 \%$ & $2.39 \%$ & $12.29 \%$ \\
\hline $1920-1929$ & $15.70 \%$ & $1.02 \%$ & $2.39 \%$ & $3.41 \%$ & $19.11 \%$ \\
\hline $1930-1939$ & $23.21 \%$ & & $1.37 \%$ & $1.37 \%$ & $24.57 \%$ \\
\hline $1940-1949$ & $20.14 \%$ & $1.02 \%$ & $3.07 \%$ & $4.10 \%$ & $24.23 \%$ \\
\hline $1950-1959$ & $12.29 \%$ & $1.02 \%$ & $0.68 \%$ & $1.71 \%$ & $13.99 \%$ \\
\hline Total & $84.98 \%$ & $3.41 \%$ & $11.60 \%$ & $15.02 \%$ & $\begin{array}{r}100 \% \\
(293)\end{array}$ \\
\hline
\end{tabular}

Nota: el valor absoluto total se ofrece en la última columna, última línea, entre paréntesis. Con él se puede realizar fácilmente el cálculo del valor absoluto al interior de cada casilla.

Fuente: Cálculos propios. Estadísticas vitales, 1885-1955.

Para el Distrito sur de Quintana Roo se observa un patrón interesante donde los padres exógamos conforme van pasando los años de nacimientos de los hijos primogénitos, hasta el decenio 1930-1939, va aumentando su proporción hasta alcanzar 23.21\%. A partir de 
este quinquenio la proporción disminuye hasta llegar a 12.29\% de toda la muestra (Cuadro $5)$.

Cuadro 6. Cambio de endo/exogamia de los padres a través del tiempo de generación de nacimiento del hijo registrado en el Distrito de Corozal.

\begin{tabular}{|c|r|r|r|}
\hline $\begin{array}{c}\text { Decenio de nacimiento } \\
\text { del hijo registrado }\end{array}$ & \multicolumn{1}{c|}{$\begin{array}{c}\text { Padres } \\
\text { exógamos }\end{array}$} & $\begin{array}{c}\text { Padres endógamos } \\
\text { de Corozal }\end{array}$ & Total \\
\hline $1880-1889$ & & $1.41 \%$ & $1.41 \%$ \\
\hline $1890-1899$ & $2.82 \%$ & & $2.82 \%$ \\
\hline $1900-1910$ & $4.23 \%$ & $8.45 \%$ & $12.68 \%$ \\
\hline $1910-1919$ & $7.04 \%$ & $9.86 \%$ & $16.90 \%$ \\
\hline $1920-1929$ & $30.99 \%$ & $25.35 \%$ & $56.34 \%$ \\
\hline $1930-1939$ & $4.23 \%$ & $5.63 \%$ & $9.86 \%$ \\
\hline Total & $49.30 \%$ & $50.70 \%$ & $100 \%$ \\
$(71)$ \\
\hline
\end{tabular}

Nota: el valor absoluto total se ofrece en la última columna, última línea, entre paréntesis. Con él se puede realizar fácilmente el cálculo del valor absoluto al interior de cada casilla.

Fuente: Cálculos propios. Estadísticas vitales, 1885-1955.

El comportamiento de los padres endógamos tanto de los quintanarroenses como de los corozaleños dentro del Distrito sur de Quintana Roo es un tanto errático, ya que se mantiene ligeramente constante con niveles más bajos para los primeros, con porcentajes entre $0.34 \%$ y $1.02 \%$ en los decenios señalados para el primogénito. Mientras que para los padres endógamos de Corozal no hay una tendencia clara en sus porcentajes señalados (Cuadro 5).

Para los nacimientos ocurridos dentro de los límites del Distrito de Corozal hay un comportamiento a través del tiempo algo más claro tanto para los padres exógamos como para los endógamos, específicamente de Corozal, no hay de Quintana Roo. Para el registro 
del nacimiento del primogénito de los padres exógamos éste va aumentando hasta el decenio de 1920-1929 con 30.99\%, y el siguiente decenio baja a 4.23\%. En cuanto a los padres endógamos sucede la misma tendencia, es decir, a la alza el porcentaje de parejas hasta llegar a $25.35 \%$ en el decenio $1920-1929$, y baja a $5.63 \%$ en el último decenio (Cuadro 6).

Si bien entonces el comportamiento es similar tanto para padres endo y exógamos en el Distrito de Corozal a través del tiempo, hay una diferencia y ésta estriba en que hay más padres endógamos que exógamos en términos generales (Cuadro 6).

\section{A MANERA DE CONCLUSIONES}

El proceso de sociabilidad que se llevó a cabo durante, por lo menos, los primeros treinta años del siglo XX ejerció un efecto sobre las dinámicas demográficas muy interesantes en esta región de estudio. Sobre todo cuando se analiza a esta población segmentada por características de clase y etnia, a través del constructo de culturas íntimas. En primer lugar, uno de los aspectos a resaltar es que las diferentes localidades del norte de Belice, evaluadas a partir de los datos de nacimientos y defunciones del Distrito de Corozal, presentan una dinámica más continua, y hasta podríamos decir con una característica de una población estable — nacimientos y defunciones constantes a través del tiempo-, mientras que para las localidades del sur de Quintana Roo tienen tasas de crecimiento realmente bajas, pero en aumento constante a través del tiempo (Cuadro 1).

Las Tasas Globales de Fecundidad así lo avalan. De hecho en la selección de mujeres establecidas para el estudio muestran un constante aumento de ellas. Sin embargo, la reproducción a ambos lados de la frontera es diferencial, pues para el caso de las mujeres seleccionadas al sur de Quintana Roo tienen ligeramente tardío su primogénito, y menores TGF, sobre todo para los primeros decenios, en comparación que las del Distrito de Corozal. Ello denota la disrupción en los nacimientos de los hijos de estas mujeres, causado por la separación de los esposos o parejas, o el deseo de retrasar la maternidad hasta después de 
moverse. Este mecanismo se ha observado comúnmente en migrantes temporales, y se puede denotar que la fecundidad es menor entre los migrantes que en los no migrantes (Chattopadhyay et al. 2006, Lindstrom y Giorguli 2002).

Estas características diferenciales en torno a su reproducción, es decir, la ausencia de la convergencia de los patrones demográficos al interior de la región de estudio, se observan entre los diferentes grupos sociales - culturas íntimas-. La cultura íntima Dominante retrasa sus nacimientos, sobre todo para las del sur de Quintana, tiene bajas TGF, mientras que el grupo de la cultura íntima Residual A, que escenifica o representa la dinámica general de toda la región, presenta las mayores tasas de fecundidad y empieza tempranamente a tener hijos, pero sobre todo, las diferencias están más marcadas cuando se le compara entre territorios, donde las mujeres de Corozal, sus indicadores muestran mayor fecundidad que las del sur de Quintana Roo. Si continuamos en la misma lógica es muy posible que ello se deba a primero nuevas y precarias condiciones materiales de vida, segundo retraso de los nacimientos por efecto de disrupción familiar causado por la migración y, por último, un mercado matrimonial restringido y constreñido por factores de clase y de etnia, lo que favorece la exogamia (Esteve y Cabré 2004, United Nations 1988).

La formación de las familias de esta región no se presenta de manera igualitaria a ambos lados de la frontera. Por un lado, en el sur de Quintana Roo se ha privilegiado la exogamia, sobre todo de gente proveniente fuera de la región de estudio. Para la cultura Dominante, sin embargo, ello no es una opción, pues se observan bajos porcentajes de exogamia, y en la cultura íntima Residual A la exogamia es muy común. Mientras que en Corozal, hay altos porcentajes de endogamia, y ello se observa también para las culturas íntimas analizadas aquí. Ahora bien, se observan cambios en la relación exo/endogamia, sobre todo para el sur de Quintana Roo, lo que nos denota cambios en los patrones reproductivos sobre todo ligados posiblemente a cambios en la estructura y composición promovida por la migración, y muy posiblemente a la asimilación/integración a los lugares destino (De Valk et al. 2004, Thorvaldsen 2005). Es decir, conforme la gente, que tiene un origen étnico 
diverso pero símil en cuestión de clase, va entablando relaciones de sociabilidad a través del tiempo y se van enlazando relaciones de alianza - matrimoniales, económicas - y poder entre ellos, lo que fueron primero relaciones exogámicas van cambiando a endogámicas, sobre todo en las clases dominantes.

Los migrantes internos hacia Quintana Roo, así como los internacionales, primordialmente de Corozal y Orange Walk, provenían de ambientes muy parecidos a los del sur de Quintana Roo, lo que les permitía insertarse fácilmente en las actividades productivas, de hecho ya contratados desde antes de que llegaran a su lugar de destino. Socioeconómicamente hablando se estaban abriendo en los primeros decenios del siglo XX nuevos nichos de mercado, promovido directa o indirectamente por los asentamientos de trabajadores enganchados así como por los presidiarios que llegaban a Quintana Roo (Higuera 2002). Por ende, y a pesar de una política de la élite del gobierno nacional adversa hacia la inmigración con miras hacia el asentamiento definitivo ésta no pudo ser realmente controlada, dado los intereses por parte de la élite local que buscaba a toda costa tener la suficiente fuerza de trabajo para satisfacer la demanda de un mercado internacional que requería los productos de la selva quintanarroense (Macías Richard 1997b).

La forma de administrar Quintana Roo fue modificándose con el cambio de la élite del gobierno nacional, por un gobierno revolucionario con tintes populares. Un efecto de estos cambios, vigente hasta nuestros días, es la aparición en el escenario de nuevas formas de tenencia de la tierra, como son los ejidos y los pequeños propietarios, y éstos subcontratarían la explotación de sus recursos (César y Arnaiz 1983). En cuanto a la pequeña propiedad privada tiene su origen en el primer decenio del siglo XX, en el reparto de pequeños solares destinados a labradores pobres, aumentando su distribución desde el decenio de los años 30 para declinar a finales de los 70, con respecto a los arrendamientos y las concesiones (César y Arnaiz 1983, Macías Richard 1997a). 
Estos cambios de política en las élites nacional y local pudieron producir cambios significativos en el proceso migratorio de Quintana Roo, atrayendo más la migración interna que la internacional. Estos nuevos inmigrantes se insertaban dentro del esquema propuesto de ejido y de la pequeña propiedad privada llegando hasta el decenio de los años setenta y ochenta con los grandes proyectos de colonización del sureste del país (Fort 1979: 209).

Es de señalar que la inmigración hacia Quintana Roo fue de tipo familiar, donde se asentaba a ambos lados de la frontera habilitando campos sociales trasterritoriales, trasnacionales, dando como resultado una causación acumulativa de la migración, forjando redes y flujos continuos de migrantes; para posteriormente, decenios después integrarse a la sociedad mexicana evaluado aquí a través de los patrones de endo/exogamia en como registraban a sus hijos.

La historia del norte de Belice, vista a través de la dinámica demográfica del Distrito de Corozal, muestra tan sólo la consolidación de la estructura social y demográfica que se venía gestando desde por lo menos 50 años antes del siglo XX, con la llegada de los refugiados de la guerra de castas a estas latitudes de Honduras Británica (Dobson 1973). Así se muestra la consolidación de un mercado matrimonial balanceado, propiciado por una inmigración posiblemente familiar de otras partes del mundo, un crecimiento natural sostenido gracias a estabilidad de la población; las estructuras sociales y culturales se encontraban bien establecidas a partir de fronteras, casi inamovibles, claras al interior de la sociedad beliceña, divididos los individuos por su pertenencia a una clase y una etnia, lo que promovía la asimilación al sistema colonial inglés, perpetuándose las diferencias de clase que se venían gestando desde el siglo XIX.

Consecuentemente, en la región de estudio existió una migración tanto dirigida, es decir, organizada por un gobierno central (presidiarios, operarios) como espontánea, bajo iniciativa de las familias e individuos que buscaban nuevas formas de subsistencia. Esta 
migración está circunscrita a un contexto de hegemonía local y nacional, para el caso de Quintana Roo y colonial para Honduras Británica, que promovió y limitó, a través de los años, la inmigración a la región, lo cual modificó la estructura de la población a ambos lados de la frontera. En Quintana Roo hubo un claro desbalance en el mercado matrimonial e impulsó diferentes comportamientos en la formación de parejas, dependiendo del stock de gente casadera tanto por edad, sexo y grupo social. Una vez establecidas las parejas, sus comportamientos reproductivos fueron diferentes dependiendo a qué grupo social y generación pertenecían las parejas que formaron estas familias, promoviendo el crecimiento social y económico del sur de Quintana Roo a principios del siglo XX.

Mientras que en Belice, los condicionantes eran otros y solamente se consolidaba como una importante puerta de entrada hacia México para los migrantes internacionales que recibía. Por consiguiente, Belice vivió y vive una mayor integración a México debido a una historia compartida, mientras que con el resto de Centroamérica y el Caribe se percibe alejada y muchas veces antagónica. Y, en la actualidad, no hay gran diferencia con el siglo pasado, donde la actividad comercial y demográfica tiene su segundo respiro en esta franja trasfronteriza de México-Belice.

\section{FUENTES DE CONSULTA}

Aguirre, Amado, Alberto Zenteno, Salvador Toscano, Juan de Dios Rodríguez, Rafael López Ocampo, Gregorio M. Ávalos y J. Guillermo Freyman, 1925, Informe que rinde al C. Presidente de la República el Jefe de la Comisión nombrada por el mismo, para hacer el estudio del: Territorio Federal de Quintana Roo, Imprenta de la Dirección de Estudios Geográficos y Climatológicos, Tacubaya, D. F., México.

Alba, Richard y Reid M. Golden, 1986, «Patterns of ethnic marriage in the United States», Social Forces, v. 65, n. 1, The University of North Carolina Press, North Carolina, pp. 202223. 
Alba, Richard y Victor Nee, 1999, «Rethinking assimilation theory for a new era of immigration», en The handbook of international migration: the American experience, editado por Hirschmann, Charles, Philip Kasinitz y Josh DeWind, Russell Sage Foundation, New York, pp. 137-160.

Arnaiz Burne, Stella M., A. Miranda Olán, J. A. Hoy Manzanilla, Ligia A. Sierra Sosa, Bonnie L. Campos Cámara, Leidy C. Hernández Trueba, y M. E. Cauich Piña, 1993, Estudio integral de la frontera México-Belice, Tomo II, Monografías de México, Centro de Investigaciones de Quintana Roo, Chetumal, Quintana Roo.

Basch, Linda, Nina Glick Schiller, y Cristina Szanton Blanc, 1994, Nations unbound: transnational projects, postcolonial predicaments and deterritorialized Nation States, Gordon and Breach, Amsterdam.

Bolland, O. Nigel, 1992, Colonialismo y resistencia en Belice. Ensayos de sociología histórica, Consejo Nacional para la Cultura y las Artes, Editorial Grijalbo, México.

Bourdieu, Pierre, 1976, «Marriage strategies as strategies of social reproduction», en Family and society. Selections from the annales economies, sociétiés, civilisations, editado por Forster, Robert y Orest Ranum, The Johns Hopkins University Press, Baltimore and London, pp. 117-144.

Cal Rosado, Ángel Eduardo, 1992, «Belice, Joven Nación», Cultura Sur, año IV, v. 2, n. 17, Conaculta, México, pp. 3-8.

Casillas, Rodolfo y Manuel Ángel Castillo, 1994, Los flujos migratorios internacionales en la frontera sur de México, Secretaría del Trabajo y Previsión Social, Consejo Nacional de Población, México. 
César Dachary, Alfredo y Stella Maris Arnaiz Burne, 1983, Estudios socioeconómicos preliminares de Quintana Roo. Sector agropecuario y forestal (1902-1980), Centro de Investigaciones de Quintana Roo (CIQRO), Puerto Morelos, Quintana Roo, México.

Chattopadhyay, Arpita, Michael J. White, y Cornelius Debpuur, 2006, «Migrant fertility in Ghana: selection versus adaptation and disruption as causal mechanisms», Population Studies, v. 60, n. 2, Population Investigation Committee, London, pp. 189-203.

De Valk, Helga, Aart C. Liefbroer, Ingrid Esveldt, y KèneHenkens, 2004, «Family formation and cultural integration among migrants in the Netherlands», Genus, v. 60, n. 34, Università degli Studi di Roma «La Sapienza», Roma, pp. 9-33.

Dixon, Ruth B., 1971, «Explaining cross-cultural variations in age at marriage and proportions never marrying», Population Studies, v. 25, n. 2, Population Investigation Committee, London, pp. 215-233.

Dobson, Narda, 1973, A History of Belize, Longman Caribbean, London.

Esteve, Albert y Anna Cabré, 2004, «Marriage squeeze and changes in family formation: Historical comparative evidence in Spain, France and United States in XXth Century», Annual Meeting Program of Population Association of America 2004, Centre d'Estudis Demogràfics.

Fábregas Puig, Andrés, 1990, «El concepto de frontera. Teoría y práctica», Cultura Sur, año II, v. 1, n.7, Conaculta, México, pp. 11-15.

Fábregas Puig, Andrés, 1992a, El concepto de región en la literatura antropológica, Cuadernos Ocasionales, Gobierno del estado de Chiapas, Consejo Estatal de Fomento a la 
Investigación y Difusión de la Cultura, Instituto Chiapaneco de Cultura, Tuxtla Gutiérrez, Chiapas, México.

Fábregas Puig, Andrés, 1992b, «Repensando la frontera sur», Cultura Sur, año IV, v. 1, n. 19, Conaculta, México, pp. 4-5.

Fleury, Michel y Louis Henry, 1985, Nouveau manuel de dépouillement et d'exploitation de l état civil ancien, Troisième édition, INED, París, Francia.

Fort, Odile, 1979, «La colonisation agricole au Mexique dans l'état du Quintana Roo», Cahiers des Amériques Latines, v. 19, l'IHEAL et du CREDA, Paris, pp. 205-214.

Gans, Herbert J., 1992, «Second-generation decline: scenarios for the economic and ethnic futures of the post-1965 American immigrants», Ethnic and Racial Studies, v. 15, n. 2, Routledge, Oxford, pp. 173-192.

Gans, Herbert J., 1999, «Toward a reconciliation of "Assimilation" and "Pluralism": The interplay of acculturation and ethnic retention», en The handbook of international migration: the American experience, editado por Hirschmann, Charles, Philip Kasinitz y Josh De Wind, Russell Sage Foundation, New York, pp. 161-171.

Gledhill, John, 1999, «El reto de la globalización: reconstrucción de identidades, formas de vida trasnacionales y las ciencias sociales», en Fronteras fragmentadas, editado por Gail Mummert, El Colegio de Michoacán, CIDE, Zamora, Michoacán, pp. 23-54.

Gordon, Milton, 1964, Assimilation in America life: the role of race, religion, and nationals origins, Oxford University Press, New York. 
Guarnizo, Luis Eduardo y Michael Peter Smith, 1999, «The locations of transnationalism», en Transnationalism from below, editado por Michael Peter Smith y Luis Eduardo Guarnizo, Transaction Publishers, New Brunswick and London, pp. 3-34.

Henry, Louis, 1983, Manual de demografía histórica, Editorial Crítica, Barcelona, España.

Higuera Bonfil, Antonio, 2002, Quintana Roo entre tiempos. Política, poblamiento, explotación forestal, 1872-1925, 2a. edición, Universidad de Quintana Roo, Instituto Quintanarroense de la Cultura, México.

Hubbard Urrea, Enrique, 2000, Belize, vecino ignorado. La perspectiva de un Embajador de México, Universidad de Quintana Roo, Chetumal, Quintana Roo.

Kearny, Michael, 1996, Reconceptualizing the peasantry: anthropology in global perspective, Westview, Boulder.

Kuntz Ficker, Sandra, 2007, El comercio exterior de México en la era del capitalismo liberal, 1870-1929, El Colegio de México, México.

Lindstrom, David P. y Silvia Giorguli Saucedo, 2002, «The short- and long term effects of U.S. migrant experience on mexican women's fertility», Social Forces, v. 80, n. 4, The University of North Carolina Press, North Carolina, pp. 1343-1370.

Locoh, Thérèse, 1996, «Les facteurs de la formation des couples», en Démographie: analyse et synthèse. Causes et conséquences des évolutions démographiques, Centre français sur la population et le développement. Dipartimento di Scienze Demografiche. Università degli Studi di Roma «La Sapienza», Paris, France, pp. 49-88. 
Lomnitz-Adler, Claudio, 1995, Las salidas del laberinto. Cultura e ideología en el espacio nacional mexicano, Joaquín Mortiz, Planeta, México.

Macías Richard, Carlos, 1997a, «La colonización insular y costera en el Caribe mexicano (1884-1902)», Revista Mexicana del Caribe, año II, n. 3, Universidad de Quintana Roo, México, pp. 112-151.

Macías Richard, Carlos, 1997b, Nueva frontera mexicana: milicia, burocracia y ocupación territorial en Quintana Roo (1902-1927), Colección Sociedad y Cultura en la Vida de Quintana Roo III, Consejo Nacional de Ciencia y Tecnología, Universidad de Quintana Roo, México.

Massey, Douglas S., 1985, «Ethnic residential segregation: a theoretical synthesis and empirical review», Sociology and Social Research, v. 69 n. 3, University of Southern California, Los Angeles, pp. 315-350.

Massey, Douglas S. y Nancy A. Denton, 1988, «Suburbanization and segregation in U.S. metropolitan areas», The American Journal of Sociology, v. 94, n. 3, University of Chicago, Chicago, pp. 592-626.

Ortega Muñoz, Allan, 2006, «Cultura regional: un marco teórico para el estudio del proceso de trasterritorialismo en los asentamientos de la región Quintana Roo-Honduras Británica (Belice), 1900-1935», en Itinerarios. Cultura, memoria e identidades en América Latina y El Caribe, compilado por José Luis González y Franco Savarino, INAH, ENAH PROMEP, México, pp. 175-199.

Portes, Alejandro, Luis E. Guarnizo, y Patricia Landolt, 1999, «The study of transnationalism: pitfalls and promise of an emergent research field», Ethnic and Racial Studies, v. 22, n. 2, Routledge, Oxford, pp. 217-237. 
Portes, Alejandro y Min Zhou, 1993, «The new second generation: segmented assimilation and its variants», Annals of the American Academy of Political and Social Science, v. 530, pp. 74-96.

Thorvaldsen, Gunnar, 2005, «Marriage and names among immigrants to Minnesota», en http://mcel.pacific.edu/jahc/Thorvaldsen/ThorIndex.html [consulta: 4 de junio de 2005].

Toussaint Ribot, Mónica, 1988, «La relación México y Belice: el reclamo territorial a la solidaridad de la Independencia», Nuestra América, año VIII, n. 23, UNAM, México, pp. 121-135.

United Nations, 1988, First marriage: patterns and determinants, United Nations, Department of International Economic and Social Affairs, New York.

Vallarta Vélez, Luz del Carmen, 2001, Los payobispenses. Identidad, población y cultura en la frontera México-Belice, Colección Sociedad y Cultura en la Vida de Quintana Roo, Universidad de Quintana Roo, Consejo Nacional de Ciencia y Tecnología, Chetumal, Quintana Roo, México.

\section{NOTAS}

\footnotetext{
${ }^{1}$ Generalmente se usa el intervalo protogenésico que es el espacio de tiempo que media entre la fecha de casamiento o unión de los padres y la fecha de nacimiento del primogénito. Sin embargo, este dato no se tiene por lo que se decidió usar una medida proxi que es la fecha de nacimiento de la madre, la cual sí se tenía gracias a la reconstrucción familiar y la fecha del primogénito.
}

Fecha de recepción: 11 de septiembre de 2012.

Fecha de aceptación: 7 de enero de 2013. 